\title{
BMJ Barriers to, and facilitators in, introducing Open integrated diabetes care in Ireland: a qualitative study of views in general practice
}

\author{
Sheena Mc Hugh, ${ }^{1}$ Monica O'Mullane, ${ }^{2}$ Ivan J Perry, ${ }^{1}$ Colin Bradley ${ }^{3}$
}

To cite: Mc Hugh S,

O'Mullane M, Perry IJ, et al. Barriers to, and facilitators in, introducing integrated diabetes care in Ireland:

a qualitative study of views in general practice. BMJ Open 2013:3:e003217. doi:10.1136/ bmjopen-2013-003217

- Prepublication history for this paper is available online. To view these files please visit the journal online (http://dx.doi.org/10.1136/ bmjopen-2013-003217).

Received 13 May 2013 Revised 28 June 2013 Accepted 1 July 2013
${ }^{1}$ Department of Epidemiology \& Public Health, University College Cork, Cork, Ireland ${ }^{2}$ Department of Public Health, Faculty of Health Care and Social Work, Trnava University, Trnava, Slovakia ${ }^{3}$ Department of General Practice, University College Cork, Cork, Ireland

Correspondence to Dr Sheena Mc Hugh; s.mchugh@ucc.ie

\section{ABSTRACT}

Objective: To examine the barriers to, and facilitators in, improving diabetes management from the general practice perspective, in advance of the implementation of an integrated model of care in Ireland.

Design: Qualitative using semistructured interviews.

Setting: Primary care in the Republic of Ireland.

Participants: Purposive sample of 29 general practitioners (GPs) and two practice nurses.

Methods: Data were analysed using a framework approach.

Results: The main barriers and facilitators occurred at the level of the health system but had a ripple effect at an organisational, professional and patient level. The lack of targeted remuneration for diabetes management in the Irish health system created apathy in general practice and was perceived to be indicative of the lack of value placed on chronic disease management in the health system. There were 'pockets of interest' among GPs motivated by 'vocational' incentives such as a sense of professional duty; however, this was not sufficient to drive widespread improvement. The hospital service was seen as an essential support for primary care management, although some participants referred to emerging tension between settings. The lack of coordination at the primary-secondary interface resulted in avoidable duplication and an 'in the meantime' period of uncertainty around when patients would be called or recalled by specialist services. Facilitators included the availability of nursing support and serendipitous access to services. The lack of resources in the community was considered to be at odds with policy to shift routine management to general practice, which is fast reaching saturation.

Conclusions: At present, intrinsic motivation is driving the improvement of diabetes care in Ireland. This will not be sufficient to implement the proposed reform including a national model of integrated care.

Policymakers need to assess and prepare for the disparate levels of interest and infrastructure in primary care in Ireland to support this change.

\section{INTRODUCTION}

In 1982, an article in the British Medical Journal suggested that the "care of [people

\section{ARTICLE SUMMARY}

Article focus

- Over the past two decades, most health systems have reoriented type 2 diabetes care from acute reactive services to regular integrated management in the primary care setting.

- In Ireland, there are plans to reorganise and standardise diabetes care through the introduction of a national model of integrated care whereby the regular management of uncomplicated type 2 diabetes would shift to primary care.

- In advance of this reform, the aim of this study was to identify the current barriers to, and facilitators of, integrated diabetes care from the general practice perspective.

\section{Key messages}

- The main barriers to integrated diabetes care were at the level of the health system, including a lack of remuneration for chronic disease management in general practice and difficulties in coordinating care across the primary-secondary interface. These barriers had repercussions at an organisational, professional and patient level.

- Efforts to improve diabetes care relied on vocational incentives and serendipitous access to services.

- There was a strong opinion that policy proposals to shift routine management to primary care needed to be supported by adequate resources and investment in community services.

Strengths and limitations of this study

- The use of qualitative methods allowed us to understand the views of the healthcare professionals expected to be part of the implementation and maintenance of integrated diabetes care on the ground.

- This study represents the views of those working in the general practice setting. It is equally important to understand the perspective of those in specialist secondary care services and to elicit patients' preferences regarding the organisation of diabetes care.

with diabetes] requires enthusiasm and organisation." ${ }^{\text {The authors endorsed }}$ general practice as the setting for the 
management of uncomplicated diabetes, working closely with hospital specialists. Most developed countries have since reoriented care from episodic management in the acute setting to regular structured management in the primary care setting, in recognition of the growing diabetes epidemic and the changing needs of patients.

The chronic care model, which is frequently cited as a guiding framework for policy and reform in this area, proposes major changes to the organisation and delivery of care for people with chronic illnesses. This model highlights a number of essential components for highquality care including self-management support, delivery system redesign, decision support and clinical information systems. Community resources, including links with other healthcare professionals, are another component of effective care. ${ }^{23}$ Diabetes is particularly challenging to coordinate given the myriad of healthcare providers and settings involved. National and international emphasis is now on integrated care which focuses on the organisation of management within settings and the coordination of care between settings. ${ }^{4-6}$

In Ireland, the introduction of integrated diabetes care is one of the pillars of the National Clinical Care Programme for Diabetes, a programme established to improve the quality of care and patient outcomes. Under this model, the routine management of uncomplicated type 2 diabetes will shift to primary care while those with complicated type 2 diabetes will be managed between primary and secondary care. ${ }^{5}$ These changes are set against a backdrop of wider health system reform including the proposed introduction of free GP services for people with chronic conditions as part of a move towards a universal health insurance model. Currently in Ireland, some people are entitled to free acute GP care under the General Medical Scheme based on means testing (37\% of the population eligible in 2011). ${ }^{7}$ Those who are not eligible must pay to attend the GP. In terms of diabetes care, at present there are a variety of care arrangements in place across the country including traditional hospital-based management, shared care between general practitioners (GPs) and hospitals, primary care-led management ${ }^{89}$ and unstructured opportunistic care. The most recent survey of diabetes management in general practice highlighted the lack of formal integration between settings. ${ }^{10}$ Furthermore, there was a lack of structured management within the practice and insufficient access to services; over $30 \%$ of GPs did not have direct access to dietetic services while more than $40 \%$ did not have direct access to podiatry services.

Recent studies, which have examined the implementation of integrated chronic disease care in countries such as England and Denmark, have emphasised the importance of context. ${ }^{11}{ }^{12}$ The aim of this study was to examine the barriers to, and facilitators in, delivering integrated diabetes care from the general practice perspective, in the light of proposed reforms in the Irish health system. Healthcare professionals are key to the success or failure of improvement initiatives depending on their willingness to learn, accept and adapt to changes in practice ${ }^{13}{ }^{14}$; therefore, it is imperative to understand their perspectives prior to implementation.

\section{METHODS}

This qualitative research was part of a wider study examining the organisation of diabetes care in Ireland, barriers to, and facilitators of, optimal management and attitudes to quality improvement initiatives including the establishment of a national diabetes register. This paper focuses on themes which emerged in relation to the first and second research objectives.

\section{Participants and sampling}

The study population consisted of GPs working in the Republic of Ireland who had 'opted in' during a preceding postal survey on the organisation of diabetes care. There was a $44 \%$ response rate to the initial survey $(n=262)$ and the majority of participants indicated a willingness to be contacted for a follow-up interview $(n=213$, $81 \%$ of survey respondents). ${ }^{10}$ In total, 25 of 26 counties in the Republic of Ireland were represented in the study population and the profile of the survey respondents was broadly comparable with the national GP profile in terms of the urban/rural breakdown and proportion of singlehanded/group practices. ${ }^{15}$ Approximately half of the original survey respondents self-reported a special interest in diabetes, and most practices had a computer system (93\%) in line with the national profile of general practice. ${ }^{15}$

Purposive sampling was used to capture the diversity of experiences in general practice. Participants were selected from practices which varied in size, urban/rural location and degree of practice computerisation, factors which could influence the organisation of care (table 1). A total of 31 interviews were conducted with 29 GPs and two practice nurses nominated by the practices as the lead healthcare professional responsible for diabetes. Participants were initially contacted by letter and followed up by telephone contact during which the researchers explained the study aims and methodology. Each participant received an information sheet and outline of the topic guide in advance of the interview.

\section{Interviews}

Face-to-face interviews were conducted between July 2009 and January 2010, all of which took place in the

\begin{tabular}{lcl} 
Table 1 & Participant matrix $(\mathrm{N}=31)$ & \\
\hline & Urban (16) & Rural (15) \\
\hline Single (15) & 6 & 9 \\
Computerised & 4 & 8 \\
Non-computerised & 2 & 1 \\
Group (16) & 10 & 6 \\
Computerised & 10 & 6 \\
Non-computerised & 0 & 0 \\
\hline
\end{tabular}


participant's practice or home. Two researchers conducted the interviews $(\mathrm{SMH}=16, \mathrm{MOM}=15$ interviews) using the same semistructured topic guide, which was informed by the results of the aforementioned national survey and two pilot interviews. The topic guide was organised around five key issues: how diabetes care is currently delivered in the practice, challenges to managing diabetes effectively and efficiently, changes in care provision at the local and national levels, attitudes to the introduction of quality improvement strategies and participants' 'wish list' for diabetes care in Ireland. Prompts and probes were used throughout the interviews to stimulate discussion. Signed informed consent was obtained before each interview, which lasted $50 \mathrm{~min}$ on average. All but three of the interviews were digitally recorded and transcribed verbatim. Interviews which were not recorded at the request of participants were typed up from extensive field notes.

\section{Analysis}

Data were analysed iteratively, that is concurrently with data collection, to allow emerging themes to be explored in subsequent interviews. ${ }^{16}{ }^{17}$ Findings from the initial interviews were discussed by the multidisciplinary research group which included expertise in health services research, social policy, epidemiology and public health and general practice (SMH, MOM, IJP, CB). Discussions led to the reformulation of some of the prompts used during the interview. A checklist was originally devised to encourage the interviewee to indicate whether a factor was a barrier to, or facilitator of, care delivery in his/her practice. However, experience during the interviews and initial analysis suggested that this format was restrictive and superficial as participants considered certain factors to be barriers as well as facilitators depending on their presence or absence in the practice. Hence, the checklist was modified into a written prompt, around which participants could discuss their views and experiences.

The framework approach was used for data analysis. ${ }^{17}$ This method enabled investigation of a priori objectives identified in the topic guide while also allowing new themes to emerge from the data. The systematic analytical process also provided transparency, which facilitated analysis and discussion by multiple researchers. ${ }^{18}$ Initially, interview transcripts were analysed independently by each researcher (SMH and MOM). The transcripts were read repeatedly (familiarisation) and themes and concepts were identified. A thematic index or conceptual framework was developed from these codes based on the key areas of the topic guide, which also included newly emerging themes. In this study, it was decided not to apply the index to the data as the data were already quite orderly given the semistructured nature of the interview schedule. ${ }^{17}$ Data were sorted and synthesised by theme, bringing similar concepts together (thematic charting). Throughout the analysis, the language and expressions of the GPs were maintained as far as possible to avoid losing the meaning and context.

Following separate first wave analysis, the researchers examined the convergence and divergence of their findings. Divergence arose from two conditions: (1) different labels or codes applied to the same concept or (2) unique concepts emerging from a researcher's analysis not identified by the other researcher. Overall, a similar 'constellation of themes' were identified and the difference lay in the labels applied to those themes, that is, 'packaging' as described in another study employing multiple analysts. ${ }^{19}$ Through discussion, it became clear that divergent codes typically related to the same concept and consensus was reached on the most appropriate label to apply. Occasionally, unique codes emerged from one researcher's analysis, which were discussed with the wider group. Three interviews from each researcher were subject to inter-coder reliability by an independent party not involved in data collection but familiar with the design and aims of the study (CB).

Memo writing was also used as an analytical tool to identify avenues for analysis and interpretations to pursue, connections or comparisons between data and to uncover the assumptions of the participant as well as the researcher. ${ }^{20} \mathrm{~A}$ summary of our interpretation was sent to each participant for respondent validity purposes. None of the participants requested changes to the interpretation of the data.

\section{RESULTS}

The participants in this study represented the diversity of diabetes care arrangements in Ireland. There was variation in the level of organisation around diabetes care within practices from 'ad hoc' opportunistic management to structured diabetes care characterised by regular recall and review, patient registration and nurse coordination. Although most participants were from computerised practices, the extent to which they utilised electronic records varied. There were also different experiences of sharing care with hospital specialists. Hence, barriers and facilitators were rooted in the context of care delivery; a particular support or resource could be a barrier or facilitator depending on its presence or absence in a participant's practice.

Barriers and facilitators occurred at multiple levels within the health system and had knock-on effects. The main barriers to optimal management occurred at the health system level: lack of remuneration for diabetes management, lack of coordination between settings and deficient access to services, particularly in the community. Facilitators included the availability of a practice nurse or diabetes nurse specialist, and serendipitous access to services which participants felt was due to good 'luck' rather than a process of service planning in the health system. Figure 1 illustrates these barriers and facilitators and where they occur within the health system. The figure was developed based on analysis of the 


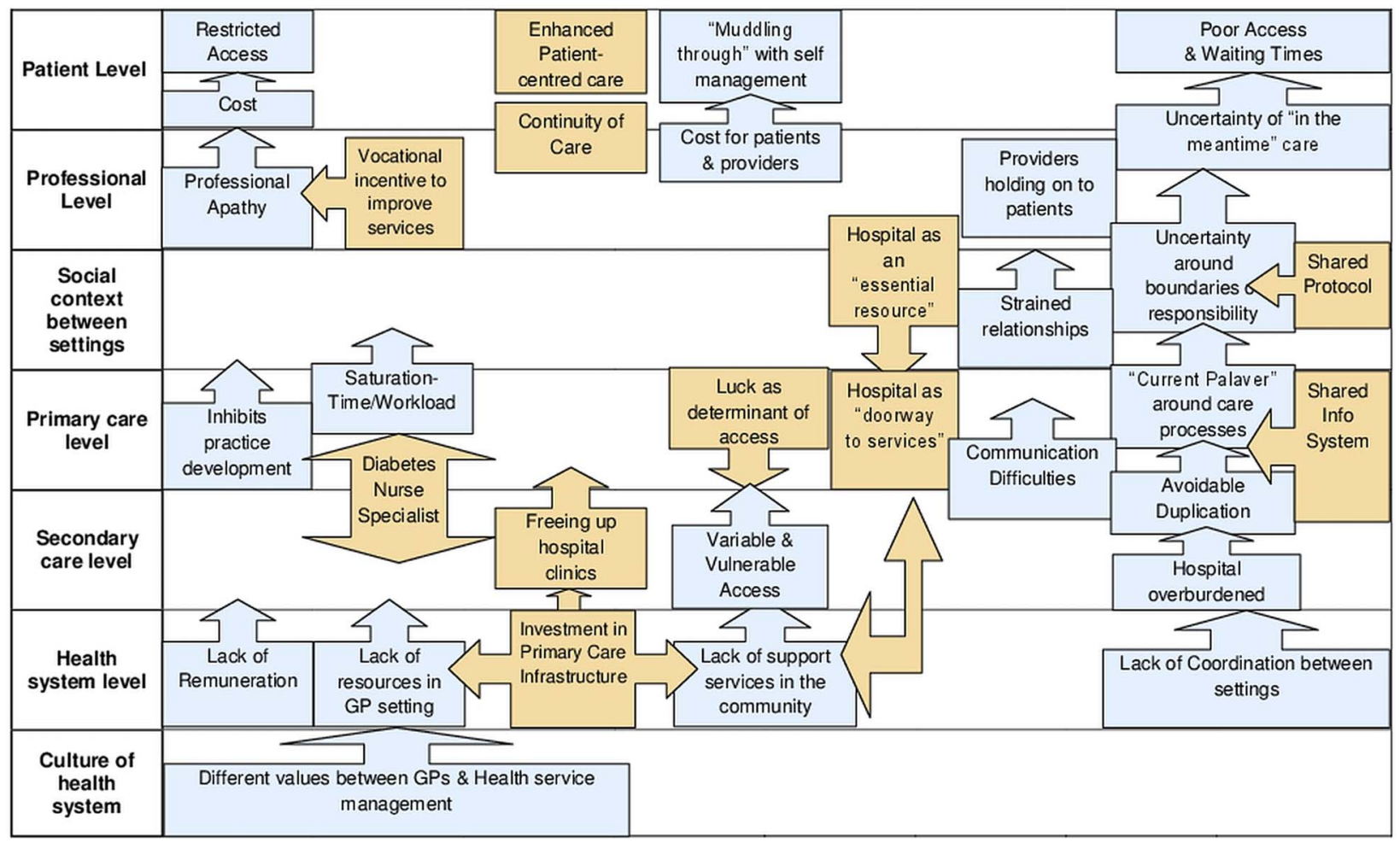

Figure 1 Barriers to, and facilitators in, delivering integrated diabetes care. barrier

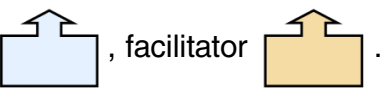

transcripts. The connections identified within the diagram are based on the ramifications of various barriers as identified by participants themselves during the interview process.

\section{Financial disincentives}

The lack of targeted remuneration or financial incentives to provide structured diabetes management in general practice emerged as one of the main barriers. The 'non-existent' remuneration was a barrier to care delivery across single physician and group practices in urban as well as rural areas. Current forms of remuneration left those practices that provided regular structured diabetes care at 'a financial loss'. Practices are paid an annual capitation grant to cover the cost of providing acute services to patients eligible under the General Medical Scheme. This funding is not linked to the intensity or quality of care provided, which could act as 'a disincentive' to do more, according to some participants. Despite the flaws of the current system, there were divided opinions about the most appropriate form of remuneration. Participants expressed concern about performance-based remuneration and the potential to 'corrupt' the provision of care and its providers. One participant voiced the concern that "once you incentivise it [diabetes management] other things that you can't incentivise get lost or diminished in the process."

The lack of remuneration led to barriers at other levels of the system including stunted practice development, imposing a cost barrier on patients without a medical card and creating a sense of apathy and 'bad feeling' among GPs (box 1). Some participants related the lack of remuneration to the underlying differences in the priorities and values of healthcare professionals and management in the health system. There were frequent references to 'us' and 'them' in relation to policymakers and health service management. One participant

\section{Box 1 Consequences of the lack of remuneration}

- Cost implications for patients without a medical card "If we're getting no recognition and no incentive and no remuneration or anything to do this work, l'd be mad in the head to... keep doing it unless I charge the patient and I don't like doing that but I don't have any choice." (GP101, rural single-handed practice)

- Impeding practice development at an organisational level "At the moment care is opportunistic but if there were incentives for me to hold a clinic that would help. We could keep flow charts and l'd get remuneration because there is none and this [work] takes a lot of time, manpower, secretarial time, nurse time, and at the moment there's no incentive to do that." (GP5, urban group computerised practice)

- Professional apathy

"There's no real recognition for it, which is important, because think that if things do come down on us, things like [administering] the flu vaccine, which is a minor point, we'll sort of say 'fine', but it'll show apathy and then it'll be 'okay, we'll see our diabetics once a year now', because we have to end up making money elsewhere." (GP112, rural group computerised practice) 
suggested it was "typical of this health system that [the] things we value highly we don't get paid for, so it's really [down to] your own interest". Others called for a new contract which recognised general practice as the most efficient and economical place for managing chronic conditions. However, the lack of resources in the community was seen as a barrier to shifting chronic disease management from the acute setting. Participants described how "they [policy makers] want us to do everything in the primary care setting which is understandable... as much as possible it would be nice to be supported to be able to do that but it would mean more staff and that inevitably brings in things that would limit staff which would be incentives and remuneration."

\section{'Vocational' incentives}

Participants made the distinction between the financial and 'vocational' incentives for providing regular structured diabetes care. 'Vocational' incentives related to a sense of personal or professional obligation to improve care delivery, which acted as an internal motivation for some participants (box 2). Those who referred to vocational incentives as a source of motivation had established a systematic structured approach to diabetes management, either as part of a local primary care

\section{Box 2 Vocational incentives}

- Job satisfaction

"If we can get the older diabetics some better control, we'd be doing some good...so from a personal, job satisfaction professional point of view that would be a huge incentive for me" (GP110, rural group computerised practice)

- Patient feedback

"We're starting people on insulin who really need it and they feel so much better. [Patients say] 'my energy's back up'...so it's very rewarding, you get a lot of good feedback from patients" (GP103, urban group computerised practice)

- Personal experience

"My own father was in hospital 7 or 8 years ago. He was on the ward and there were 7 other guys, it was a vascular ward, and they were all diabetics. Some of them were in for 8,12 weeks, or 16 weeks....incredible. At the same time I read somewhere that if you can get, is it, a $1 \%$ drop in the $\mathrm{HbA1C}$, reduces the complications by $25 \%$.So that struck a chord with me..." (GP110, rural group computer practice)

- Professional duty

"...the international evidence is such that we felt duty bound to offer as tight control as we can for patients" (GP103, urban group computerised practice)

- Personal values and priorities

"Depending [on] what your attitude is and your enthusiasm [for] preventative stuff, which to me is just as important if not more important and it's enjoyable" (GP101, rural single computerised practice)

"We feel that we've improved the service that they've [patients] been given, so that was the incentive, but that's the only incentive" (GP112, rural, group computerised practice) initiative or independently within their own practice. However, participants acknowledged that a special interest in diabetes was limited to "pockets' of practices and "you can't expect all GPs to be desperately keen on it." Beyond this group, remuneration and financial incentives would be the main facilitator to providing structured care in the practice and therefore the biggest barrier to engaging all GPs at present.

\section{Lack of integration between settings and professionals}

The relationship between practices and secondary care diabetes teams was largely positive for most participants who described the hospital-based specialist service as 'a resource', 'an essential support' and a 'doorway to services'. A small number of participants reported an unconstructive relationship with the hospital-based team, which was a barrier to delivering optimal care. These participants felt it would be hard to take part in integrated care 'with the power struggle between primary care and secondary services'. Part of this struggle related to attempts on both sides of the primarysecondary interface to 'hold on' to patients.

According to participants, the primary barriers to integrated care did not occur at the professional level but at the level of the health system. The lack of coordination within the system manifested itself in a number of ways and had ramifications in both settings (box 3). In the first instance, participants expressed uncertainty about the boundaries of responsibility and highlighted the need for "clear definition as to what the hospital is going to do, [and] what we're expected to do." Most participants wanted joint involvement between consultants and GPs rather than an either/or situation which had negative implications for the patient and the professional according to one participant: "if you only deliver care in acute services then people are left floundering for 6 or 9 months in between...but equally if you only see them in the community and they don't have a link with the hospital, when they run into a problem its sometimes very difficult to get somebody in quickly because they're not part of the system."

In addition, the lack of coordination and integrated management led to a sense of ambiguity around the patients' care pathway. A number of participants referred to an 'in the meantime' period; this referred to uncertainty about when patients would be called or recalled by specialist services. For example, one participant from an urban single-handed computerised practice 'supposed' "that we're to pick them [patients] up in the meantime," that is between hospital reviews, but "there's been no communication, there's been no meetings, there's been no working group... it's just sort of left like that." Another participant from an urban group practice described how patients with diabetes were recalled "by the system...as the hospital deems appropriate" with problems referred to the hospital in the meantime, "that's as much of a protocol as there is." This sense of uncertainty around the care pathway also 


\section{Box 3 Symptoms of the lack of coordination}

- Uncertainty of 'in the meantime care' for patients and providers

"If you could say to [patients] 'look this is your diagnosis, this is what we're going to discuss and over the next 2 weeks you're going to meet $A, B, C$ and then we have a baseline of everything covered from day 1 and you know exactly where you are, you're on a springboard ready to jump. As opposed to... saying 'stand on the spring board for about 2 months and then 2 months later you might get called for your eyes and 2 months later you might be called for your feet', in which time they may have had a problem with their feet and they're not quite sure how they should have dealt with it..." (Practice Nurse 104, rural group practice)

- 'Overburdened' hospital clinics and waiting times

"I notice that they're pushing them [reviews] out further and further, the reviews would have been 6 months some time ago...

its gone to 2 years...Consultants just don't have time, they can't see everybody. It's just not possible. They're doing their best. l've no complaints about their service at all." (GP113, urban singlehanded practice)

"There's a massive diabetic clinic in the hospital but care is not better. It's difficult to make appointments, get access to services, especially when it's urgent." (GP10, urban single-handed practice)

- The lack of boundaries and bureaucratic 'palaver' around processes of care

"At the moment it's a big palaver if you check cholesterol, get it to the patient to bring into the hospital and it gets lost in the process lots of times and then it seems incredibly wasteful of effort and time and resources." (GP106, urban group practice)

"...patients spend the last precious days of their lives going from out-patients to out-patients, confused as to who to believe, and in the ideal world, the GP service would be coordinating and making sure it doesn't happen too much...then they get lost in the follow-up, it gets so complicated" (GP108, rural group practice)

\section{- 'Avoidable duplication'}

"Unfortunately they still have to be seen in the hospital annually because for things like retinopathy screening and podiatry care, there isn't one single unit where you could refer them...they have to go through the clinic, there's a bit of duplication that could be avoided" (Practice Nurse 104, rural group practice).

emerged in relation to accessing support services (illustrated by the quote in box 3) and was seen as particularly difficult for patients who were newly diagnosed with diabetes. Furthermore, there was concern that some patients are 'falling through the net' and not attending either service.

Participants called for the development of a shared protocol to reduce avoidable duplication and clarify the roles and responsibilities within each setting. Those from computerised practices emphasised the need for shared information systems to minimise duplication and overcome the current 'palaver' surrounding processes of care, such as routine blood tests, in the system. Participants from non-computerised practices did not view non-computerisation as a barrier to integrated care or optimal diabetes management.
It's what works for me. I'm updated in terms of training and meetings and all that kind of thing...But I would like to see us having a place in anything that would develop [in terms of the implementation of integrated diabetes care] (GP115, rural single-handed non-computerised).

\section{Support services-'Not enough of them and too hard to access'}

Participants described access to support services such as dietetics, podiatry and retinopathy screening as "nonexistent' and 'abysmal' in some areas. The lack of services in the community and the resultant reliance on hospitals for the management of 'finer details like eyes and feet' was a barrier to community-based diabetes care. While the hospital was 'a doorway to services' for some, others described the secondary care services as a 'fortress' with lengthy waiting lists. Where services were in place in the community, availability was frequently described as 'patchy' in urban as well as rural areas. Access to services such as dietetics and podiatry fell along a scale from good to bad, or bad to worst in some cases. Few participants had access to a complete range of services for their patients. The availability of services was further jeopardised by the ongoing government policy to freeze recruitment in the public sector due to the economic recession. As a result, maternity leave was not covered and those who retired were not replaced. One participant from an urban practice described how "foot care is not particularly good, it's a bit random...Dieticians were good, we had a community dietician and then she went on maternity leave and she wasn't replaced so now again it's a bit patchy." The most significant impact was on patients who were left 'muddling through'. One participant described a patient "who had very poor eyesight due to diabetes and we couldn't get a community podiatrist to see her... at all. I mean it [the waiting list] was about 2 years or something they told me...she wasn't a priority."

\section{'Lucky' to have access to specialists and support services}

Several participants referred to themselves and their patients as 'lucky' to have access to services such as dieticians and podiatrists, suggesting that these services evolved by chance rather than systematic service planning within the health system. Participants acknowledged the unique position they were in given the aforementioned 'patchy' access to services. Access in some cases resulted from the resourcefulness of healthcare professionals in establishing partnerships or optimising opportunities.

\footnotetext{
We're very lucky here, in that we've had a very good access to a dietician for the last few years now. It was just something they tried themselves. They were based in the city, and they decided to put some outreach clinics out in the county, and they picked this town for one of their centres.
}

Participants also referred to luck in relation to the availability of a dedicate nurse within the practice or access to a diabetes nurse specialist. This facilitated the delivery 
of structured care within the practice and coordination between settings and specialists. Diabetes Nurse Specialists were described as 'worth their weight in gold' and 'the single best thing to happen to diabetes from the management point of view'. Improvements in quality of patient care were attributed to enhanced nurse-led services in the practice and hospital setting.

\section{Risk of general practice becoming saturated}

Time, resources and workload emerged as barriers to providing optimal diabetes care in general practice. However, participants did not dwell on these "limiting factors", which were perceived as almost inevitable. Time "is always a barrier", but timing was given more attention by participants who emphasised the importance of early intervention and regular review. Participants warned about the risk of general practice becoming saturated as more services are moved to the community, including other chronic physical and mental illnesses and the "huge amount of bloods being done" in primary care. The health system "needs to back up their policy of shifting [management] from secondary care to primary care" by providing adequate resources.

We always have been willing to take on more and more stuff that is primary care-based [and] bring it out of secondary care but we're saturated now (GP110, rural group computerised)

\section{DISCUSSION}

Although desirable, the barriers to integrated care should not be underestimated. ${ }^{21}$ The main barriers identified in this study were system-level deficiencies including the lack of remuneration for chronic disease management, poor coordination at the primary-secondary care interface and insufficient services, particularly in the community, which forced GPs to rely on the hospital as a doorway to other healthcare professionals. These challenges have a ripple effect throughout the system at an organisational, social, professional and patient level. Participants' accounts of providing 'in the meantime care' and the bureaucratic 'palaver' surrounding routine processes of care expose the lack of integration within the system currently, as well as the negative impact on efficiency, professional relationships, quality of care and patient experience.

The aim of this study was to identify and understand the barriers to, and facilitators of, optimal diabetes care from the general practice perspective, in advance of the proposed reorganisation of services in Ireland. The national model of integrated care has yet to be implemented, and therefore the results of this study provide an opportunity to anticipate future barriers and plan solutions which take into account the local context of care provision. Research from the fields of implementation science and quality improvement has focused on ways to overcome structural and contextual barriers through tailored incremental change and professional leadership. $^{1122} 23$ In this study, participants proposed shared protocols and linked information systems as mechanisms to facilitate the provision of integrated care. While the National Clinical Care Programme for Diabetes has proposed care pathways for different patient groups, the absence of a single electronic medical record and unique patient identifier complicates efforts to share information and track patient care. Appropriate investment and infrastructure is needed to foster and support widespread participation in quality improvement.

The results support the contention that "something more than personal financial gain is driving professional behaviour" (p.5). ${ }^{24}$ Vocational incentives were seen as the primary motivation for engaging in quality improvement in the absence of adequate financial remuneration. The distinction between types of incentive is congruent with existing theories of health worker motivation which identify individual, organisational and cultural determinants. ${ }^{25}$ Organisational determinants of motivation, such as communication processes and organisational support through adequate resources and efficient service delivery, are in short supply according to the participants in our study. Remuneration was considered necessary for widespread improvement as intrinsic incentives were limited to 'pockets of interest'. Policymakers deciding on the ideal payment structure for chronic disease management should take into consideration the context ${ }^{26}$ and the alignment of values between the professional and the organisation. $^{24}$ Participants commented that current $^{2}$ systems of remuneration reflected discordance between the values of the health service management and healthcare professionals in terms of the importance of investing in chronic disease management and the contribution of primary care.

Some of the themes identified, such as the part played by local circumstances and 'luck' in securing access to community services, are rooted in the context of the Irish health system and the history of underinvestment in primary care in Ireland. Despite the nuances of this system, similar barriers and facilitators have been identified in other countries. ${ }^{67-29}$ While previous studies classified factors influencing diabetes care at the level of the patient, provider and organisation/system, this study unpacked further layers of healthcare delivery, identifying barriers and facilitators relating to the relationships between providers, professional attitudes and the culture underlying the health system. A metasynthesis of the barriers and facilitators to improvement should be conducted, to develop and understand the full extent of the evidence base. ${ }^{30}$ A number of the barriers have implications beyond diabetes care and correspond to issues facing the wider primary care setting. ${ }^{31}$ Consequently, there is ample opportunity to learn from other settings and countries in terms of how to overcome these barriers.

\section{Limitations}

Participation in this study was based on an 'opt in' procedure during a preceding survey of GPs in Ireland. ${ }^{10}$ 
This could influence the profile of those who volunteered to be interviewed; however, this is a consideration for all qualitative studies. Owing to the anonymous nature of the survey, it was not possible to link practice and professional demographics to those who agreed/did not agree to be interviewed. The survey sample was in line with the national profile of general practice in Ireland in terms of practice size, location and level of computerisation. ${ }^{15}$ Participants' experiences typified the diversity of care arrangements in the Irish health system and the organisation of diabetes care in practice ranged from ad hoc to structured management. While practice characteristics guided the sampling strategy, perceptions of the main barriers were largely consistent across the subgroups and attitudes were shaped to a greater extent by the context of care in terms of access to various supports and services. The merits of involving more than one analyst in a qualitative study have been debated. ${ }^{32} 33$ In addition to the predominance of research teams in health services research, analysts from different disciplines can bring breadth and depth to the findings. ${ }^{34} 35$ Furthermore, this approach is often considered an implicit form of inter-rater reliability. ${ }^{19}$ Inter-rater reliability was also applied explicitly in our study. The technique is considered appropriate in the context of semistructured interviews whereby all participants are asked broadly the same questions in the same order ${ }^{36}$ Nevertheless, there is a risk of over-simplifying codes and themes to facilitate independent checking, and therefore inter-rater reliability was used following the first wave of analysis on initial codes to minimise this risk.

This study presents the barriers to, and facilitators of, optimal diabetes management from the general practice perspective. The other healthcare professionals involved in the delivery of diabetes care may face unique challenges within their setting or profession. Furthermore, while participants highlighted barriers within the system for patients such as the out-of-pocket costs associated GP care in Ireland, further research with patients is warranted to garner their views on the introduction of integrated diabetes care in Ireland. A similar qualitative study has been conducted on patients' attitudes to shared care arrangements in Ireland. ${ }^{37}$

\section{CONCLUSIONS}

The key ingredients of organisation and enthusiasm, highlighted in $1982,{ }^{1}$ are reflected in the constellation of themes which emerged in this study of the barriers to, and facilitators of, optimal integrated care. Given the proposals to reform diabetes services through the introduction of integrated care and the transfer of uncomplicated type 2 diabetes management to primary care, there is a need to understand the current challenges to delivery in this setting. Reorientation of care must be accompanied by the reorganisation of support and resources. Vocational incentives are motivating factors for 'pockets' of healthcare professionals; however, this is not sufficient for the widespread engagement of GPs in the delivery of a national model of integrated diabetes care.

Acknowledgements The authors would like to thank the doctors and nurses who participated in this study. This study was conducted on behalf of the National Diabetes Register Project (NDRP) group.

Contributors The design of this study was developed in close collaboration among all four authors. SMH and MO were responsible for data collection and analysis. CB acted as the inter-rater during the analysis phase. Feedback on the interpretation was received from all the authors. SMH drafted the paper for submission and all authors provided feedback and approval for the final version.

Funding This study was conducted on behalf of the National Diabetes Register Project (NDRP) group which was funded by the Health Research Board, Ireland (grant No.:HS/2006/14). SMH was also funded by the Health Research Board (HRB) as a PhD Scholar of the Health Services Research Institute, the HRB's Cross-Institution PhD Scholar Programme in Health Services Research (grant No.: PHD/2007/16).

Competing interests None.

Ethics approval Irish College of General Practitioners.

Provenance and peer review Not commissioned; externally peer reviewed.

Data sharing statement No additional data are available.

Open Access This is an Open Access article distributed in accordance with the Creative Commons Attribution Non Commercial (CC BY-NC 3.0) license, which permits others to distribute, remix, adapt, build upon this work noncommercially, and license their derivative works on different terms, provided the original work is properly cited and the use is non-commercial. See: http:// creativecommons.org/licenses/by-nc/3.0/

\section{REFERENCES}

1. Thorn $\mathrm{P}$, Watkins $\mathrm{P}$. Organisation of diabetic care. $B M J$ 1982;285:787-9.

2. Wagner EH, Austin BT, Davis C, et al. Improving chronic illness care: translating evidence into action. Health Aff 2001;20:64.

3. Bodenheimer T, Wagner EH, Grumbach K. Improving primary care for patients with chronic illness. JAMA 2002;288:1775-9.

4. Gröne O, Garcia-Barbero M. Integrated care: a position paper of the WHO European office for integrated health care services. Int $J$ Integr Care 2001;1:1-16.

5. Harkins V. A practical guide to integrated type 2 diabetes care. In: Irish College of General Practitioners, Health Service Executive, Irish Endocrine Society, eds. Dublin: Health Service Executive, 2008.

6. Johnson M, Goyder E. Changing roles, changing responsibilities and changing relationships: an exploration of the impact of a new model for delivering integrated diabetes care in general practice. Qual Prim Care 2005;13:85-90.

7. Department of Health. Health in Ireland. Key Trends 2012. Dublin: Government of Ireland, 2012.

8. Brennan C, Harkins V, Perry I. Management of diabetes in primary care: a structured-care approach. Eur J Gen Pract 2008;14:117-22.

9. Mc Hugh S, Marsden P, Brennan C, et al. Counting on commitment; the quality of primary care-led diabetes management in a system with minimal incentives. BMC Health Serv Res 2011:11:384-93.

10. Mc Hugh S, O'Keeffe J, Fitzpatrick A, et al. Diabetes care in Ireland: a survey of general practitioners. Prim Care Diabetes 2009;3:225-31.

11. Vize R. Integrated care: a story of hard won success. BMJ 2012;344: e3529.

12. Rudkjøbing $\mathrm{A}$, Olejaz $\mathrm{M}$, Birk HO, et al. Integrated care: a Danish perspective. BMJ 2012;345:e4451.

13. Berwick D, Enthoven A, Bunker J. Quality management in the NHS: the doctor's role-II. BMJ 1992;304:304.

14. Scott T, Mannion R, Davies HTO, et al. Implementing culture change in health care: theory and practice. Int J Qual Health Care 2003;15:111-18.

15. O'Dowd T, O'Kelly M, O'Kelly F, et al. Structure of general practice in Ireland: 1982-2005. Dublin: Department of Public Health and Primary Care, Trinity College Centre for Health Sciences [and] Irish College of General Practitioners, 2006.

16. Pope C, Ziebald S, Mays N. Analysing qualitative data. In: Pope C, Mays N. eds. Qualitative research in health care. 3rd edn. London: Blackwell Publishing, 2006. 
17. Ritchie J, Lewis J. Qualitative research practice: a guide for social science students \& researchers. Thousand Oaks: Sage Publications, 2003.

18. Pope C, van Royen P, Baker R. Qualitative methods in research on healthcare quality. Qual Saf Health Care 2002;11:148-52.

19. Armstrong D, Gosling A, Weinman J, et al. The place of inter-rater reliability in qualitative research: an empirical study. Sociology 1997;31:597.

20. Charmaz K. Constructing grounded theory: a practical guide through qualitative analysis. London: Sage Publications Ltd, 2006.

21. Godlee F. Integrated care is what we all want. BMJ 2012;344.

22. Berwick DM. Disseminating innovations in health care. JAMA 2003;289:1969-75.

23. Lemmens K, Nieboer A, Rutten-Van M, et al. Bottom-up implementation of disease-management programmes: results of a multisite comparison. BMJ Qual Saf 2011;20:76-86.

24. Marshall M, Harrison S. It's about more than money: financial incentives and internal motivation. Qual Saf Health Care 2005;14:4.

25. Franco LM, Bennett S, Kanfer R. Health sector reform and public sector health worker motivation: a conceptual framework. Soc Sci Med 2002;54:1255-66.

26. Gosden T, Forland F, Kristiansen I, et al. Capitation, salary, fee-for-service and mixed systems of payment: effects on the behaviour of primary care physicians. Cochrane Database Syst Rev 2000;3.

27. Agarwal G, Pierce M, Ridout D. The GP perspective: problems experienced in providing diabetes care in UK general practice. Diabet Med 2002;19(s4):13-20.
28. Brown JB, Harris SB, Webster-Bogaert S, et al. The role of patient physician and systemic factors in the management of type 2 diabetes mellitus. Fam Pract 2002;19:344-9.

29. Khunti K, Ganguli S, Baker R, et al. Features of primary care associated with variations in process and outcome of care of people with diabetes. Br J Gen Pract 2001;51:356-60.

30. Dixon-Woods M, Booth A, Sutton AJ. Synthesizing qualitative research: a review of published reports. Qual Res 2007;7:375-422.

31. Oelke ND, Thurston WE, Dipalma R, et al. Understanding issues in primary care: perspectives of primary care physicians. Qual Prim Care: Radcliffe Publishing, 2006;253-8.

32. Malterud K. Qualitative research: standards, challenges, and guidelines. Lancet 2001;358:483-8.

33. Bradley E, Curry L, Devers K. Qualitative data analysis for health services research: developing taxonomy, themes, and theory. Health Serv Res 2007;42:1758.

34. Pope C, Mays N. Qualitative research: reaching the parts other methods cannot reach: an introduction to qualitative methods in health and health services research. BMJ 1995;311:42-5.

35. Pope C, Ziebland S, Mays N. Qualitative research in health care: analysing qualitative data. BMJ 2000;320:114

36. Morse J. "Perfectly Healthy, but Dead": the myth of inter-rater reliability. Qual Health Res 1997;7:445.

37. Smith SM, O'Leary M, Bury G, et al. A qualitative investigation of the views and health beliefs of patients with type 2 diabetes following the introduction of a diabetes shared care service. Diabet Med 2003;20:853-7. 